\title{
Expertise Differences in Movement Switching in Kendo Players
}

\author{
Noriaki Usui $^{1}$, Motoki Okumura ${ }^{1}$ and Kazutoshi Kudo ${ }^{2}$ \\ ${ }^{1}$ Graduate School of Education, Tokyo Gakugei University, 4-1-1 Nukuikita-machi, Koganei-shi, Tokyo 184-8501, Japan \\ E-mail:m162210n@st.u-gakugei.ac.jp \\ ${ }^{2}$ Graduate School of Interdisciplinary Information Studies, The University of Tokyo, \\ Komaba 3-8-1, Meguro, Tokyo 153-8902, Japan \\ [Received June 29, 2017; Accepted January 30, 2018; Published online February 15, 2018]
}

\begin{abstract}
To succeed in many sports, players must not only adjust their movements during execution in response to environmental changes, but also switch to different movements altogether. Previous studies have implicated executive functions in movement switching ability. We aimed to clarify expertise differences in movement adjustment and switching, and compared performance on three tasks of target striking between expert and intermediate kendo players as participants. Task 1 was a simple strike, whereby participants struck targets at one position; Task 2 was an adjusted strike, whereby participants struck targets at three positions in a random order. Task 3 was strike-defense switching, whereby participants struck targets as in Task 2 while were also required to switch to defense when an opponent made a counter-strike. That is, the participants were required accurate strike in Task 1 and 2 close to the kendo practices, and were required accurate strike and defense in Task 3 close to the real matches. We found no differences in strike movement time and accuracy in any of the tasks between groups. However, expert players were able to execute successful strike-defense switching in Task 3, whereas intermediate players were not. These results suggest that an expertise difference exists in executive functions, and that it is essential for players to practice focused on acquisition of switching skills in order to further improve their performance.
\end{abstract}

Keywords: martial arts, cognitive and motor skills, executive function, response selection, strike-defense switching

\section{Introduction}

In many one-on-one ball games and martial arts, performance success does not solely rely on players' ability to execute pre-programed movements. Rather, players must adjust their movements during execution in response to environmental changes. For example, expert table tennis and baseball players must adjust their movements during execution to hit a ball accurately (Bootsma and van Wieringen, 1990; Ranganathan and Carlton, 2007). Moreover, expert fencing players can hit a target rapidly and accurately even when the target moves during hitting movement (Gutiérrez-Dávila et al., 2013a; 2013b). These examples indicate that expert sports players have acquired the cognitive and motor skills necessary to adjust their movements during execution.

However, players do more than adjust their movements during execution-for example, in ball games, players must switch between multi-directional hitting and running movements. In interpersonal competitions, players must frequently and rapidly switch between offensive and defensive movements within a second. Switching movements require players to rapidly execute at least two different movements in a parallel or serial fashion. Researchers have proposed that the ability to flexibly switch between movements during their execution is supported by executive functions that is distinctive from the ability to execute a single movement (Gilbert and Burgess, 2008). Especially in martial arts such as kendo and karate, players are required switching movements frequently.

In the previous studies about expertise differences in martial arts, researchers have indicated that expert karate players can anticipate and react to their opponents more rapidly and accurately than novice 
and intermediate players (Mori et al., 2002; Williams and Elliott, 1999). Also, in real kendo matches, expert players can accurately perceive the interpersonal distance while moving and can switch their stepping forward and backward movements (Okumura et al., 2012; 2017). Even in other sports and physical activities, although researchers have investigated about expertise differences at various viewpoints such as muscle activity (Sakurai and Ohtsuki, 2000) or sensorimotor coordination (Miura et al., 2011), little information about the adjustment and switching skills are available. Therefore, in the present study, we investigated the characteristics of the skills of adjusting and switching movements among kendo players, as well as how these characteristics are affected by expertise.

In this study, we asked expert and intermediate groups of university kendo players to perform three different tasks involving striking a target on an opponent's forearm (called kote). In Task 1, they executed a simple strike, whereby a target presented in a fixed position. In Task 2 , they executed an adjusted strike, whereby a target presented at three different spatial positions in a random order. Finally, in Task 3, they executed strike-defense switching, whereby they struck a target presented as in Task 2 and were also required to switch their strike into a defensive movement whenever the opponent initiated a counter-strikes toward the players' head (called men). We measured the time and accuracy of each strike and defense movement. We hypothesized that both groups-because they have considerable athletic experience-would show no differences in Task 1. However, the expert group would show higher strike velocity and accuracy for Tasks 2 and 3 because these tasks require more complex cognitive and motor skills. In addition, because the players must not only strike their opponent, but also defend their opponent's attack accurately in real kendo matches, we hypothesized that the expert group will indicate higher defense accuracy in Task 3 that is required to switch from strike to defense as matches. Our overall aims were to clarify the relationships among simple, adjusted, and switching movements as well as expertise differences in these movements.

\section{Method}

\subsection{Participants}

The male players of a kendo team at Tokyo Gakugei University participated in this experiment. The expert group comprised eight players who had participated in formal competitions of Japan and regional universities, and the intermediate group comprised eight players who had not participated in these competitions. The expert and intermediate groups had the following means (SDs) for their general characteristics: age, 19.4 (1.1) and 20.5 (0.9) years old; athletic experience, 14.4 (1.2) and 11.1 (2.2) years; kendo rank, $3.3(0.4)$ and $3.0(0.0)$ dan; height, $173.8(4.0)$ and $176.0(4.1) \mathrm{cm}$; and weight, 69.0 (4.7) and $65.1(4.3) \mathrm{kg}$, respectively. As the results of the $t$-tests, there were no significant differences in the characteristics between the two groups (age: $t(14)=2.11, p=.053, r=.49$, dan: $t(14)=$ $1.58, p=.14, r=.38$, height: $t(14)=0.64, p=.54$, $r=.17$, weight: $t(14)=1.61, p=.13, r=.40)$. However, there was a significant difference in athletic experience $(t(14)=3.41, p=.004, r=.67)$. Therefore, there was clear differences between two groups as kendo players in real matches, but the differences could not be recognized from only measuring simple characteristics such as their age, physical forms, kendo rank. In other words, we challenged to clear the subtle differences from our viewpoints.

One of the experimenters served as participants' opponent and was responsible for presenting the experimental stimuli: the strike targets (Task 1-3) and counter-strikes (Task 3). He belonged to the same team as the participants, who had also participated in formal competitions of Japan and regional universities; he was 22 years old and had 18 years of athletic experience, a kendo rank of 4 dan, and a height and weight of $177.1 \mathrm{~cm}$ and $78.6 \mathrm{~kg}$, respectively. He had the similar skill level as the expert group.

All of the participants and the opponent were in good health and continued their athletic activities during the experiment. This study was approved by the Ethics Committee of the Tokyo Gakugei University, and the experimental procedures were carried out in accordance with the approved guidelines. All participants provided written informed consent in accordance with the Declaration of Helsinki before the commencement of the experiment. 


\subsection{Experimental tasks}

Figure 1 shows the experimental setting. First, the participants decided their preferred strike start distance (Lines 1 and 2) to ensure that they kept the distance of their strikes as constant as possible within each trial. The mean (SD) distances of the expert and intermediate groups from Line 1 to the opponent's right foot were 251 (10.4) $\mathrm{cm}$ and 249 (4.3) $\mathrm{cm}$, respectively, while the distances from Line 2 were $199(6.3) \mathrm{cm}$ and $196(8.9) \mathrm{cm}$. The $t$-tests revealed no significant differences between the two groups' distances (Line 1: $t(14)=0.58, p=.57, r$ $=.15$, Line $2: t(14)=0.70, p=.50, r=.18)$.

For all of the trials, the participants took the same ready stance (chudan; see Figure 1 (1)) and stepped forward (beginning with their right foot and following with their left) from Line 1 to 2 (Figure 1 (2)). If the opponent presented the target (his right forearm; kote) at the same time as the participants' left foot reached Line 2, the participants had to strike the target (Figure 1 (3)). However, if the opponent did not present the target, the participants were required to stop without striking (Figure $1(5))^{* 1}$. In Task 3, if the opponent presented a counter-strike toward the participants' head (men) at the same time as the participants' left foot reached Line 2, participants had to defend against the counter-strike (Figure 1 (4)). For all trials, the participants were instructed to strike and defend as rapidly and as accurately as possible.

Before the study began, the opponent practiced sufficiently and synchronized the presentation timings of the targets and counter-strikes to participants' foot movements. The mean times (SDs) of the expert and intermediate groups from the participants' strike start (i.e., their right heel rising from the floor at Line 2) to the opponent's target (moving his shinai, or sword) and counter-strike (rising his right heel from the floor) presentation were 47 (7.2) and 50 (13.2) ms, respectively (see the bottom line in Figure 1). There was no significant difference in this time between the groups $(t(14)=$ $0.15, p=.89, r=.04)$. In other words, the participants had already begun strike when the opponent started presenting target and counter-strike stimuli at about $50 \mathrm{~ms}$. Additionally, the mean times (SDs) of the opponent's counter-strikes (from his right heel rising from the floor to his sword reaching the participants' head) were 168 (6.1) and

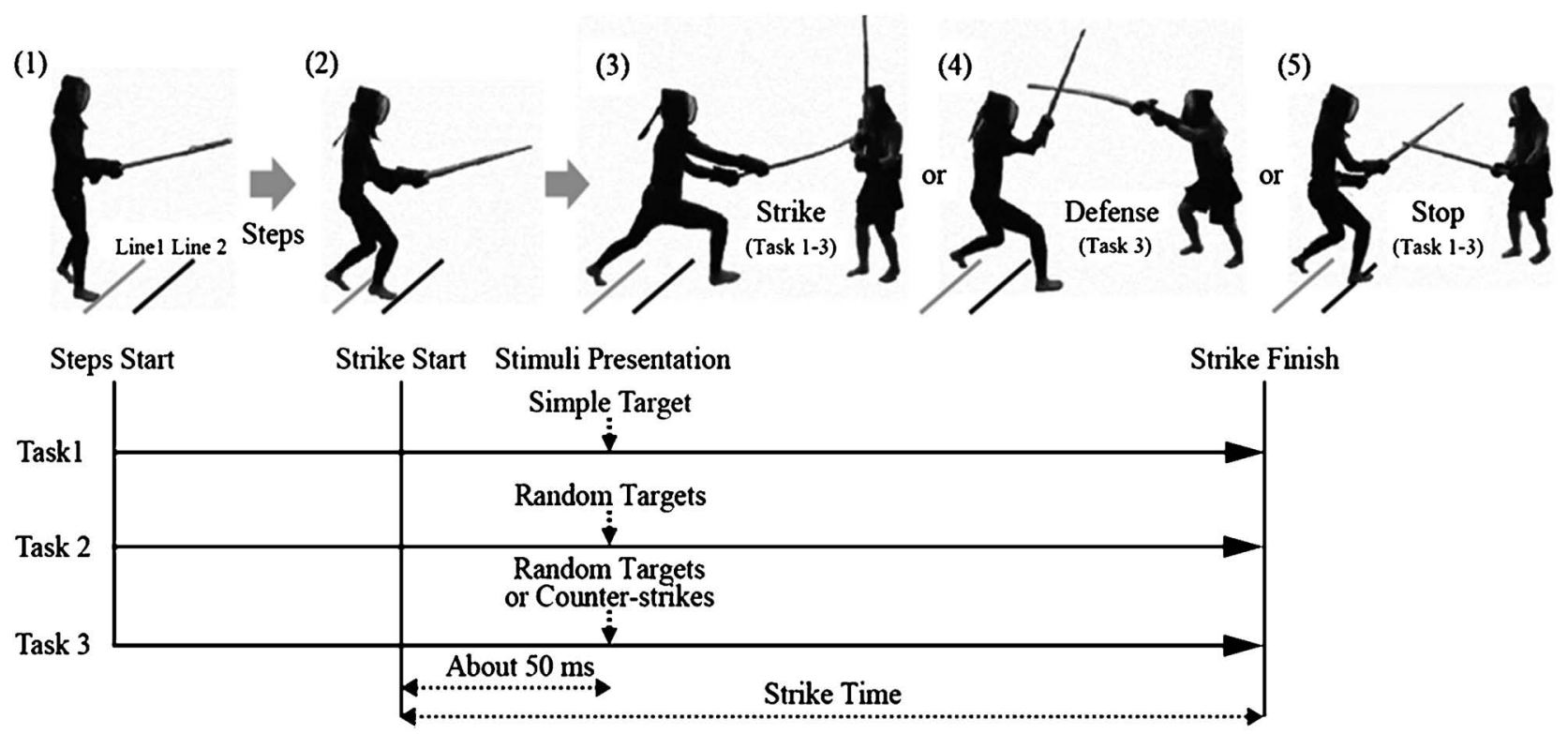

Figure 1 Experimental procedures in each task

The players were stood in a ready position at Line 1 (1) and then stepped to Line 2 (with their right first, followed by their left) (2). Then, they performed the strike in a continuous movement by stepping toward the opponent (3). The opponent presented a simple target in Task 1 and random targets at three positions in Tasks 2 and 3 (bottom lines; see Stimuli Presentation and Figure 2A). The opponent also presented counter-strikes in Task 3 (4) or did not present the target at all in every task (5). The targets and counter-strikes presented about $50 \mathrm{~ms}$ after the player started the strikes. 
$171(9.8) \mathrm{ms}$ for the expert and intermediate groups, respectively. Also, there was no significant difference between the groups $(t(14)=1.74, p=.10$, $r=.42)$.

The experiment was conducted in the same order-Task 1, 2, and 3-for all participants. The participants practiced the procedures sufficiently before beginning the experiment, took enough rest between the tasks, and never mistook the procedures in the tasks. We assumed that individual performances of the participants will not change significantly due to task order, because they frequently execute the strike adjustment and strike-defense switching in matches and practices.

\subsubsection{Task 1: Simple strike}

Task 1 was a simple strike, the participants struck at a target that the opponent presented only to the center position as shown in Figure 2A. Each participant had to judge strike or stop after starting strikes, and executed six strikes and three stops in a random order.

\subsubsection{Task 2: Adjusted strike}

Task 2 was an adjusted strike, the participants had to strike a target that the opponent presented randomly in one of three positions in Figure 2A. The left and right targets were about $10 \mathrm{~cm}$ from the center target laterally. The opponent stabilized the target positions as much as possible among all trials. Each participant had to judge strike or stop after starting strikes, and executed six strikes and three stops in a random order.

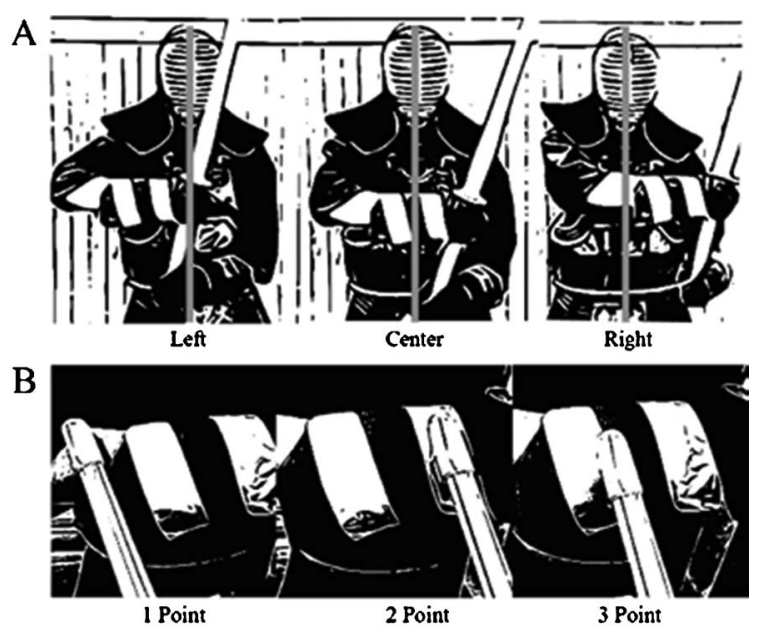

Figure 2 The target positions (A) and the scoring examples for the strike accuracy (B)

\subsubsection{Task 3: Strike-defense switching}

In Task 3, the participants had to strike a target presented as in Task 2, and in addition, had to defend whenever the opponent began a counter-strike (see Figure 1). Each participant had to judge strike, defense, or stop after starting strikes, and executed six strikes, six defenses, and three stops in a random order.

\subsection{Experimental device}

Throughout the trials, the participants' movements were recorded using a high-speed digital video camera $(240 \mathrm{frm} / \mathrm{s}$, HDR-AS100V, Sony, Inc.) placed to the left behind the participants. The camera recorded all of the necessary information, including their right feet, swords, and the targets for the following analyses.

\subsection{Data analysis}

\subsubsection{Strike time}

We measured strike time for all the tasks. We defined strike time as the time interval from participants' right heel rising from the floor at Line 2 to their sword making contact with the opponent's forearm (see Figure 1). Two experimenters analyzed the strike time. We calculated Pearson's correlation coefficient, and showed a strong correlation between the experimenters' analyses $(r=.970, p<.01)$.

\subsubsection{Strike accuracy}

We measured strike accuracy for all of the tasks. We marked the opponent's target to score the strike-specifically, we drew marks at $5 \mathrm{~cm}$ intervals from the target's central position and gave 1-3 points depending on where participants' sword tip touched (higher points were given for striking between the two marks; see Figure 2B). The participants were instructed to strike the center of the target area (which awarded 3 points) with their sword tip as rapidly and as accurately as possible by an experimenter. Two experimenters analyzed the strike accuracy. We calculated Pearson's correlation coefficient, and showed a strong correlation between experimenters' analyses $(r=.983, p<.01)$.

\subsubsection{Defense accuracy}

We measured defense accuracy in Task 3 . We found that it was difficult to confirm precisely 
whether the opponent's sword touched the participants' head, even when using the high-speed video camera. Therefore, the participants were asked to report whether they were struck by the opponent. We recorded their reports and counted the frequencies of the successful and failed defense.

\subsection{Statistical analyses}

For all statistical analyses, we used SPSS Statistics Ver. 22 (IBM Corp., Armonk, NY) and set an $\alpha$ value of 0.05 . We conducted repeated-measures two-way analyses of variance, with the 2 groups and 3 tasks as factors, on strike time and accuracy. Post-hoc comparisons were conducted using the Bonferroni method. Sphericity assumptions were verified using Mauchly's test. We also conducted Fisher's exact test to compare the frequencies of defense success or failure between the two groups in Task 3.

\section{Results}

\subsection{Strike time}

Figure 3 shows the strike time of both groups across the three tasks. Neither the main effect of group $\left(F[1,14]=0.57, p=.46, \eta_{p}^{2}=.04\right)$ nor task $\left(F[2,28]=1.29, p=.29, \eta_{p}^{2}=.01\right)$ was significant. The interaction between group and task was not significant $\left(F[2,28]=0.65, p=.53, \eta_{p}^{2}=.05\right)$.

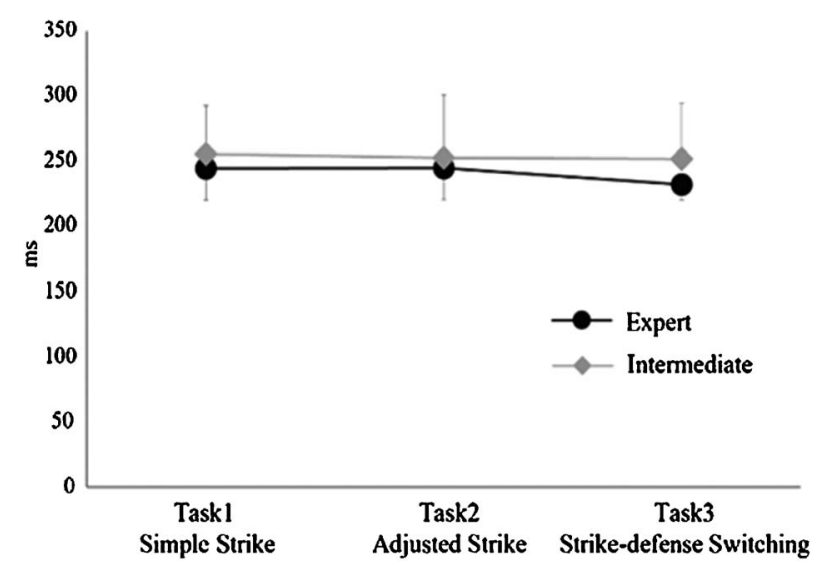

Figure 3 The strike time for the three tasks in the expert and intermediate groups

\subsection{Strike accuracy}

Figure 4 shows the strike accuracy of both groups across the three tasks. Neither the main effect of group $\left(F[1,14]=0.21, p=.65, \eta_{p}{ }^{2}=.02\right)$ nor the interaction between group and task $(F[2,28]=$ $0.05, p=.95, \eta_{p}{ }^{2}=.00$ ) was significant. However, the main effect of task was statistically significant $(F$ $[2,28]=32.41, p<.01, \eta_{p}{ }^{2}=.70$; averages $[95 \% \mathrm{Cl}]$ for Task $1=2.63[2.52,2.74]$, Task $2=2.12[1.87$, $2.36]$, and Task $3=1.57[1.29,1.84])$. Task 1 had a higher score than did Task 2, while Task 2 had a higher score than did Task 3 .

\subsection{Defense accuracy}

Figure 5 shows the average frequencies of defense success and failure for both groups in Task 3 . There was a significant difference between the groups ( $p$

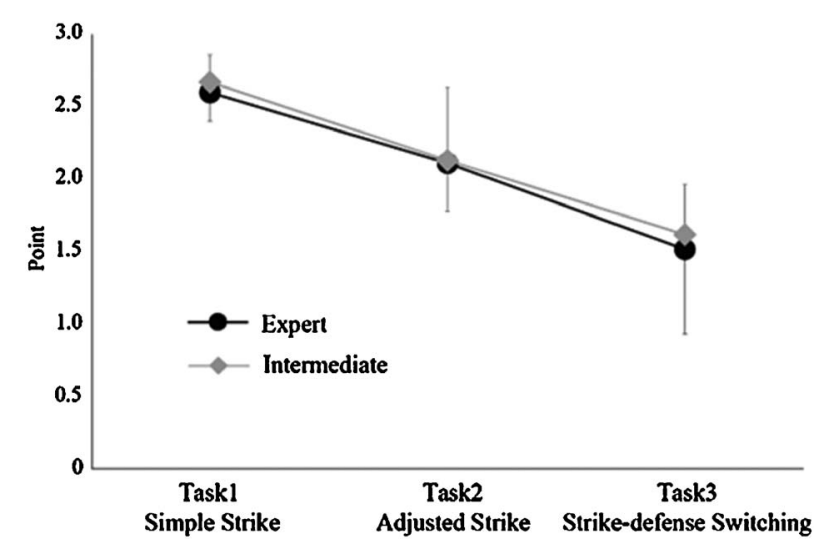

Figure 4 The strike accuracy score for the three tasks in the expert and intermediate groups

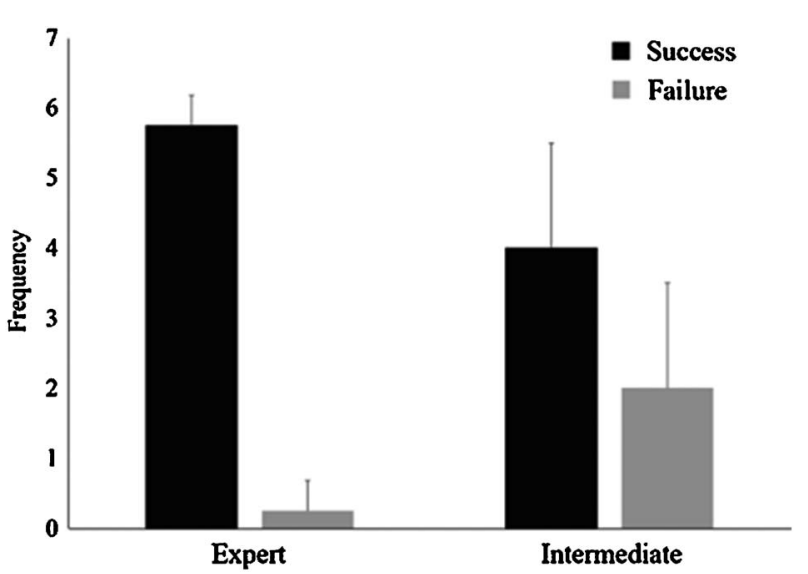

Figure 5 The frequency of defense accuracy in Task 3 in the expert and intermediate groups 
$=.0004)$. The expert group had a higher frequency of defense success (mean [SD] per 6 trials: 5.75 [0.43]) than did the intermediate group (4.00 [1.5]).

\section{Discussion}

We observed no significant differences in strike time or accuracy for Task 1 (simple strike); both groups were able to strike rapidly and accurately. In other words, both groups of participants-all of whom had about 10 years of athletic experience-had high skill in performing a simple strike.

However, contrary to our expectations, we observed no group differences in the strike time and accuracy for Task 2 (adjusted strike). Based on the experimental methods and the strike time results, the participants struck at the targets that the opponent presented in three different positions in random order and with a timing of about $50 \mathrm{~ms}$ after the participants began the strike movement. Thus, the participants had to make strike adjustments within about $200 \mathrm{~ms}$ (a strike time of $250 \mathrm{~ms}$ - a presentation time of $50 \mathrm{~ms}$; see Figures 1 and 3). While participants were able to maintain a rapid strike time in Task 2, they could not maintain strike accuracy. Previous research has similarly confirmed that expert fencing players show a $20 \%$ increase in errors when they had to rapidly strike $9 \mathrm{~cm}$ targets that were moving over a distance of about $25 \mathrm{~cm}$ compared to when the targets were not moving (Gutiérrez-Dávila et al., 2013b). These results might suggest that the players controlled strike time as an element which should be maintained even if they sacrifice strike accuracy to some extent. In kendo matches, the players must rapidly strike toward the opponent before the opponent's attack or defense movement has completed. In other words, if the player's strike time increases, the opponent could not simply defend the strike, but also counterstrike during the strike. Therefore, it was considered that the players give more priority to maintain the strike time than strike accuracy.

Also in contrast to our hypothesis, we observed no group differences in the strike time and accuracy in Task 3 (strike-defense switching). The difference between Tasks 2 and 3 was the existence of the counter-strike interference by the opponent. The participants showed lower strike accuracy in Task 3 compared to Tasks 1 and 2. The linear decline in the accuracy over Tasks 1 to 3 indicated the fact that the tasks became gradually more difficult as they progressed. Previous research has indicated that reaction time and performance failure rate increase when participants execute a reaction task such as button press responding to two stimuli compared with one stimulus (Pashler, 1994). In the sports scene, performance success rate in rugby decreases when game situations include larger number of players and high complexity (Gabbett and Abernethy, 2012); these finding and our study results demonstrate a clear relationship between lower performance and higher complexity of cognitive and motor skills.

Notably, as a support to our hypothesis, there was a significant group difference in defense accuracy in Task 3; expert group had superior skills in switching their strike into a defensive movement. Based on the experimental methods, the players had two choices of strike or stop in Task 1 and 2, and had three choices of strike, defense, or stop in Task 3 after starting strike. In Task 3 , the defense judgement of intermediate group might be delayed by the influence of choice increasing, and might cause a delay of the actual movement. In fact, according to our observations, when the opponent struck the participants' head in the task, the intermediate group did not stop their strike movement and delayed starting their defense movement. In the other hand, we could assume that the intermediate players could defend the opponent's counter-strike if they knew it in advance, because they had a defense success rate more than half trials even in the complex Task 3 (see Figure 5). Therefore, it was considered that the intermediate players have simple defense ability but not selective defense ability under multiple choices of attack and defense.

According to research in cognitive neuroscience, executive functions are located in the frontal lobe and are believed to govern switching abilities (Gilbert and Burgess, 2008). Movement switching is also considered a basic but important element of motor skills in sports science (Otsuki, 1998). Many theorists have indicated that switching abilities need to use attentional resources from single or multiple attentional pools in order to execute at least two different movements in a parallel or serial fashion, executive functions are related to allocation of attentional resources (Kahneman, 1973; Gruber and Goschke, 2004). Additionally, researchers indicated that expert fencing players demonstrated the ap- 
propriate executive function to switch rapidly and accurately from an intended movement to a new appropriate movement with stronger activity in the prefrontal lobe (Di Russo et al., 2006), and that there are expertise differences in go/no-go reaction tasks (which are believed to measure executive functions), but no differences in simple reaction tasks in the study investigating how expertise in sports influences reaction time (Kida et al., 2005). As such, based on previous studies and our results, it seems reasonable that expertise difference exist in executive functions under complex situations with multiple choice responses and that different level of executive functions are being employed between two groups.

To succeed in real kendo matches, players must not only strike but also defend their opponent's strike simultaneously. Furthermore, the results of this study suggest that switching skills are critical in kendo expertise. However, player strikes toward the opponent one-sidedly in general kendo practice. Our results suggested that it is essential for players to train focused on acquisition of switching skills in order to further improve their performance.

We believe that our findings regarding movement adjustment and switching are useful for research on various sports skills. Furthermore, the linear decline of the strike accuracy over Tasks 1 to 3 and the expertise differences in Task 3 might be helpful in understanding diverse skills and planning practices in sports. Therefore, future studies should continue seeking to understand different sports skills based on investigating cognitive and motor skills related to movement adjustments and switching, for example, by using eye and motion analyses.

\section{Conclusion}

In this study, we had expert and intermediate kendo players engage in three tasks involving a simple strike, an adjusted strike, and strike-defense switching. While we found no group differences in the strike time and accuracy of any tasks, we did observe a significant different in defense success rate during strike-defense switching. The result that the expert group had acquired better skills for switching movements suggests that there is an expertise difference in executive functions, even when significant difference was not found in simple adjustment skills between groups.

\section{Note}

*1 We added stop trials (Figure 1 (5)) to inhibit the players from anticipating strike and defense trials. Also, because the players only stopped the strike when the opponent did not move (no-go), there was no meaning as a competitive behavior and we did not analyze their movements of the stop trials.

\section{Funding}

This research was supported in part by Grant-in-Aid for Scientific Research (C) 25350757: MO and (A) 25242059: KK from the Japan Society for the Promotion of Science.

\section{References}

Bootsma, R. J. and van Wieringen, P. C. W. (1990). Timing an attacking forehand drive in table tennis. J. Exp. Psychol. Hum. Percept. Perform., 16: 21-29.

Di Russo, F., Taddei, F., Apnile, T., and Spinelli, D. (2006). Neural correlates of fast stimulus discrimination and response selection in top-level fencers. Neurosci. Lett., 408: 113-118.

Gabbett, T. J. and Abernethy, B. (2012). Dual-task assessment of a sporting skill: influence of task complexity and relationship with competitive performances. J. Sports Sci., 30: 1735-1745.

Gilbert, S. J. and Burgess, P. W. (2008). Executive function. Curr Biol., 18: R110-R114. doi: 10.1016/j.cub.2007.12.014

Gruber, O. and Goschke, T. (2004). Executive control emerging from dynamic interactions between brain systems mediating language, working memory and attentional processes. Acta. Psychol., 115: 105-121.

Gutiérrez-Dávila, M., Rojas, F. J., Antonio, R., and Navarro, E. (2013a). Effect of uncertainty on the reaction response in fencing. Res. Q. Exerc. Sport, 84: 16-23.

Gutiérrez-Dávila, M., Rojas, F. J., Caletti, M., Antonio, R., and Navarro, E. (2013b). Effect of target change during the simple attack in fencing. J. Sports Sci., 31: 1100-7.

Kahneman, D. (1973). Attention and effort. Englewood Cliffs, NJ: Prentice Hall.

Kida, N., Oda, S., and Matsumura, M. (2005). Intensive baseball practice improves the Go/Nogo reaction time, but not the simple reaction time. Cogn. Brain Res., 22: 257-264.

Miura, A., Kudo, K., Ohtsuki, T., and Kanehisa, H. (2011). Coordination modes in sensorimotor synchronization of whole-body movement: A study of street dancers and nondancers. Hum. Mov. Sci., 30: 1260-1271.

Mori, S., Ohtani, Y., and Imanaka, K. (2002). Reaction time and anticipatory skills of Karate athletes. Hum. Mov. Sci., 21: 213-230.

Okumura, M., Kijima, A., Kadota, K., Yokoyama, K., Suzuki, H., and Yamamoto, Y. (2012). A critical interpersonal distance switches between two coordination modes in kendo matches. PLoS ONE., 7: e51877.

Okumura, M., Kijima, A., and Yamamoto, Y. (2017). Perception of affordances for striking regulates interpersonal distance maneuvers of intermediate and expert players in kendo matches. Ecol. Psychol., 29: 1-22.

Otsuki, T. (1998). “Takumi” no Kagaku [Science of “Takumi'”]. Tokyo: Asakura Syoten. (in Japanese)

Pashler, H. (1994). Dual-task interference in simple tasks: Data and theory. Psychol. Bull., 116: 220-244.

Ranganathan, R. and Carlton, L. G. (2007). Perception-action coupling and anticipatory performance in baseball batting. $\mathrm{J}$. 
Mot. Behav., 39: 369-380.

Sakurai, S. and Ohtsuki, T. (2000). Muscle activity and accuracy of performance of the smash stroke in badminton with reference to skill and practice. J. Sports Sci., 18: 901-914.

Williams, A. M. and Elliott, D. (1999). Anxiety, expertise, and visual search strategy in karate. J. Sport Exerc. Psychol., 21: 362-375.

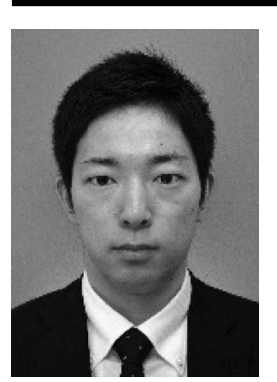

Name:

Noriaki Usui

\section{Affiliation:}

Graduate School of Education, Tokyo Gakugei University

Address:

4-1-1 Nukuikita-machi, Koganei-shi, Tokyo 184-8501, Japan

Brief Biographical History:

2016-2018 Master's Program in Graduate School of Education, Tokyo Gakugei University

Membership in Learned Societies:

- Japan Society of Physical Education, Health and Sport Sciences

- Japanese Academy of Budo 